RÉFLEXIONS

\title{
QUESTIONS POSÉES À LOUIS CHÂTELLIER, LUCE GIARD, DOMINIQUE JULIA ET JOHN O'MALLEY
}

Nous avons délibérément choisi de mobiliser, pour ce numéro, la contribution de jeunes auteurs dont l'engagement dans la recherche est contemporain du renouveau historiographique que l'on cherche ici à mieux définir. Mais il nous a semblé important de convoquer parallèlement les réflexions de quatre historiens dont les travaux et l'influence intellectuelle ont contribué à ouvrir les voies de ce renouveau, chacun dans les domaines et dans le style qui lui sont propres : Louis Châtellier, en cartographiant, au moyen d'une exploitation massive des archives de l'ordre, les relais sociaux de la Compagnie de Jésus à l'époque de la Contre-Réforme, a apporté une contribution décisive à l'histoire sociale des pratiques religieuses de l'Europe catholique; Luce Giard a défini les enjeux d'une réinscription de l'histoire de la Compagnie dans l'histoire intellectuelle de la Renaissance et placé l'interprétation des stratégies savantes de l'ordre au premier plan des recherches sur la configuration des savoirs au seuil de la modernité; les travaux de Dominique Julia donnent la mesure du rôle des collèges jésuites dans le déploiement de l'institution scolaire sous l'Ancien Régime et mettent à la disposition de la communauté scientifique des outils pour la recherche, sur une histoire que seule l'exploitation systématique des sources à laquelle il s'est livré peut faire apparaître; John O'Malley, acteur important au sein de la communauté scientifique jésuite aux États-Unis, a redécouvert l'histoire du premier siècle de la Compagnie à partir des travaux qu'il a consacrés à l'histoire de la Renaissance, sur la base desquels il a pu évaluer l'ampleur des mutations culturelles liées au développement de la Contre-Réforme catholique ainsi que la place des jésuites dans ce processus.

Nous avons proposé, à chacun de ces quatre interlocuteurs, le même questionnaire, auquel ils étaient évidemment libres de répondre selon leur choix. Qu'ils soient ici très chaleureusement remerciés de leur attentive collaboration. 


\section{LES QUESTIONS}

1) L'historiographie de la Compagnie de Jésus s'est profondément renouvelée dans les dix dernières années. Quels sont, d'après vous, les traits saillants de ce renouvellement?

2) Comment, selon vous, ce renouvellement s'inscrit-il dans le cadre des courants actuels de l'histoire du phénomène religieux?

3) Comment discernez-vous l'influence de ce renouvellement dans le champ de l'histoire du monde moderne?

4) Quels peuvent être, selon vous, les apports spécifiques d'une histoire des ordres religieux, et singulièrement de la Compagnie de Jésus, dans cette nouvelle configuration?

5) Quelles vous paraissent être la ou les spécificités de la Compagnie de Jésus par rapport à d'autres ordres contemporains dans l'histoire de cette période, et dans notre compréhension de cette histoire?

6) Les développements récents de l'historiographie jésuite ont privilégié le premier siècle. Comment analysez-vous cette tendance et concevez-vous la pertinence d'une extension de cette historiographie au xvII' siècle, voire à l'histoire de la «nouvelle Compagnie »?

7) Quels vous paraissent être les enjeux de la redécouverte, dans l'historiographie récente, d'un espace européen de la Compagnie de Jésus, par rapport aux cadres nationaux de l'historiographie « officielle » du $\mathrm{XIX}^{\mathrm{e}}$ siècle?

8) Nous vous avons demandé de répondre à ce questionnaire, car votre apport nous paraît important dans les recherches actuelles sur l'histoire de la Compagnie de Jésus. Comment définiriez-vous vous-même cet apport?

9) Pour les dix prochaines années, quels chantiers et quels problèmes vous semble-t-il devoir être poursuivis, ouverts et discutés en priorité ? 


\section{Louis Châtellier}

\section{Réponse à la question 6}

Ne s'intéresser qu'au premier siècle de l'histoire de la Compagnie revient, me semble-t-il, à avaliser une vision schématique selon laquelle les jésuites furent un produit du « temps des réformes » et que, celui-ci écoulé, ils avaient perdu leur raison d'être. Leur suppression en 1773 (bref Dominus ac Redemptor de Clément XIV) relèverait alors d'une simple opération chirurgicale consistant à couper un membre mort de l'Église. Deux raisons doivent inviter l'historien à mettre en doute ce jugement pour le moins hâtif. La première se trouve dans les Constitutions et la correspondance d'Ignace de Loyola ${ }^{1}$. Cette congrégation bâtie pour s'adapter à toutes les sociétés et aux différentes cultures aurait-elle été incapable de faire face à la nouvelle situation issue de la paix de Westphalie et des courants de pensée qui se développèrent en Europe tant dans le domaine philosophique que scientifique? Il y a là de quoi surprendre. La seconde raison repose sur des faits que l'on peut regrouper sous deux rubriques.

En premier lieu, il convient de rappeler que débuta, à partir de 1660, ce qu'on nomme, depuis quelques années, « la seconde réforme catholique ». Elle se manifesta, en particulier, par de grandes missions qui, parfois, concernèrent des pays entiers. Elles étaient organisées depuis Rome avec, pour relais, les princes ou les évêques. Or, les artisans les plus actifs de cette entreprise, tantôt de contre-réforme au sens propre du terme, tantôt purement apostolique, furent les jésuites. Leur organisation centralisée, leur expérience et surtout leur implantation dans toute l'Europe où depuis un siècle ils avaient multiplié les résidences, les collèges, les maisons professes et les universités constituaient autant de raisons solides qui amenaient les autorités civiles et religieuses à leur confier la tâche d'enraciner, parmi les populations, l'enseignement et les pratiques de piété catholiques. Le P. Paolo Segneri, au cours de ses nombreuses missions en Italie du Nord et à travers les États pontificaux, dans la seconde moitié du XvII ${ }^{e}$ siècle, mit au point le modèle qui devait servir de référence à ses successeurs tant sur le plan technique que spirituel. Il fut repris jusqu'au milieu du $\mathrm{xI}^{\mathrm{e}}{ }^{\mathrm{e}}$ siècle par les différents ordres missionnaires. Des équipes de pères jésuites

1. Ignace de Loyola, Écrits, trad. du latin et éd. sous la dir. de Maurice Gullani, Paris, Desclée De Brouwer, 1991. 
allèrent l'implanter, dès les années 1715-1720, en Suisse, dans les États de la Maison d'Autriche (Autriche, Hongrie, Bohême, Silésie, Pays balkaniques, Pays-Bas), en Bavière et en Franconie, dans l'Allemagne rhénane, le nord et l'est de la France, en Espagne et au Portugal et dans l'Italie du Sud. Ces missions ne consistaient pas seulement en de simples passages de prédicateurs pendant huit ou quinze jours dans un lieu. Par leurs retours périodiques, par les livres de piété distribués et les dévotions instituées, elles contribuèrent largement à faire évoluer la vie religieuse des fỉdèles ${ }^{2}$. Le rapide succès du culte du Sacré-Cour dont l'importance pour le catholicisme moderne a été soulignée par Mario Rosa ne se comprend pas sans l'action des jésuites ${ }^{3}$. Les PP. Pedro Calatayud en Espagne, Philippe Jeningen en Allemagne, Claude de La Colombière et Croiset en France en furent les très actifs propagandistes par la parole et par le livre. D'autres furent les premiers à prendre en compte la famille comme cellule de la vie chrétienne grâce aux confréries de l'Agonie du Christ puis, au milieu du xvıII ${ }^{\mathrm{e}}$ siècle, des nouvelles confréries de la Doctrine chrétienne ${ }^{4}$. Autant d'initiatives qui furent à la source du renouveau catholique du $\mathrm{xIx}^{\mathrm{e}}$ siècle.

Le second fait concerne l'activité intellectuelle des jésuites au Siècle des Lumières. La comparaison avec les débuts de la Compagnie conduit généralement les auteurs à conclure au déclin. Il faut dire que les apparences vont dans ce sens. Lors des événements qui conduisirent au bannissement des jésuites de France, ce ne furent pas seulement les avocats et les procureurs du roi qui relevèrent les carences de leur enseignement, mais aussi de nombreux ecclésiastiques. Ils insistèrent sur son caractère routinier, inadapté au monde moderne, en particulier pour les langues vivantes et les sciences ${ }^{5}$. Ces carences étaient d'autant plus visibles qu'au milieu du $\mathrm{XVIII}^{\mathrm{e}}$ siècle les jésuites devaient faire face à la concurrence des oratoriens en France, des bénédictins ou des chanoines réguliers en Allemagne ${ }^{6}$. Mais

2. Louis ChÂTELUER, La Religion des pauvres. Les missions rurales en Europe et la formation du catholicisme moderne, $x V^{e}-x X^{e}$ siècle, Paris, Aubier, 1993.

3. Mario RosA, " "Regalità e douceur " nell' Europa del '700. La contrastata devozione al Sacro Cuore ", Dai Quaccheri a Gandhi. Studi di storia religiosa in onore di Ettore Passerin d'Entrèves, Bologne, Il Mulino, 1988, p. 71-98; repr. et développé in ID., Settecento religiosó. Politica della ragione e religione del cuore, Venise, Marsilio, 1999, p. 17-46.

4. Annick et Louis ChâtelLIER, « Les premiers catéchistes des Temps modernes. Confrères et consœurs de la Doctrine chrétienne aux xvi'-XvII ${ }^{e}$ siècles ", in La Religion de ma mère. Le rôle des femmes dans la transmission de la foi, dir. Jean Delumeau, Paris, Cerf, 1992, p. 287-299.

5. Ainsi Louis de La Chalotais, in Essai de l'éducation nationale, Paris, 1763, dont les critiques et les suggestions furent reprises par de nombreux réformateurs des années 17601770 .

6. Marie-Madeleine COMPÈre et Dominique JuLia, Les Collèges français, $x v f^{f}-x v I f$ siècle, 2 vol., Paris, Éd. du Centre national de la recherche scientifique/Institut national de la recherche pédagogique, 1984 et 1988; pour l'Allemagne, Richard VAN DÜLMEN, "Antijesuitismus und katholische Aufklärung in Bayern ", Historisches Jahrbuch, t. LXXXIX, 1, 1969 , p. 52-80. 
les structures de la vie intellectuelle dans l'Europe des Lumières n'étaient plus celles du XvI siècle. Le collège n'en était plus le foyer rayonnant. La science se pratiquait désormais dans les académies, les observatoires, dans les premiers laboratoires (Lavoisier). Quant à ses résultats, ils étaient diffusés beaucoup plus grâce aux revues savantes que par l'enseignement des professeurs. Or, les jésuites avaient leur place dans ces lieux nouveaux où le travail scientifique était réalisé et discuté. L'examen des actes de l'Académie des sciences et de l'Académie des inscriptions et belles-lettres, au cours des premières années du XvII' siècle, le montre à l'évidence. Qu'il s'agisse de critique historique, de chronologie, d'astronomie, de géologie, d'arithmétique ou d'algèbre, les travaux et les observations des pères étaient constamment cités ${ }^{7}$. On songe, bien évidemment, à la masse d'informations qu'ils ont été capables de rassembler en Chine et dont ils firent bénéficier des savants tels que l'astronome Jean-Dominique Cassini, le philosophe mathématicien Gottfried Wilhelm Leibniz ou l'historien Nicolas Fréret ${ }^{8}$. Si ces derniers se souciaient tant de correspondre avec les membres de la Compagnie de Jésus, ce n'était pas seulement à cause des informations inédites qu'ils en pouvaient obtenir, mais, surtout, parce que recueillies par d'autres savants, elles étaient susceptibles d'être analysées avec une plus grande exactitude. Puis, ces relations savantes, dues au hasard des rencontres ou au réseau des correspondants, prirent un caractère plus cohérent. Ainsi naquirent, en 1701, les Mémoires pour l'histoire des Sciences et des Beaux-Arts, dits Mémoires de Trévoux dont le maîtred'œuvre et le directeur pendant plus de trente ans fut le P. René Joseph de Tournemine ${ }^{9}$. Grâce aux cahiers de cette revue, les savants de la Compagnie et ceux qui lui étaient extérieurs trouvaient non seulement un lieu d'expression - ce qui existait déjà avec notamment Le Journal des savans à Paris ou les Acta eruditorum à Leipzig - mais aussi une possibilité de confronter leurs idées avec celles d'autres savants dans une perspective qui était celle du catholicisme posttridentin. Il est important de constater que, dès les premiers numéros de l'année 1701, s'engagea un débat de fond avec, pour protagonistes, Jacques Bernoulli et Gottfried Wilhelm Leibniz d'une part, le P. René Joseph de Tournemine d'autre part. Il portait sur

7. Bibliothèque nationale de France, département des Manuscrits, Manuscrits français 9429, table des procès-verbaux de l'Académie royale des inscriptions et belles-lettres, $\mathrm{f}^{\mathrm{s}} 146^{-}$ 209 , catalogue des ouvrages de 1701 à 1712 ; Histoire et mémoires de l'Académie royale des sciences (de Paris), voir les volumes pour les années 1701 à 1710.

8. Mémoires de l'Académie royale des sciences (1666-1699), t. VIII (œuvres diverses de Jean-Dominique Cassini); Mémoires de Trévoux, janvier 1704, p. 128-165 (« extrait d'une lettre écrite à Mr. Leibnits par le P. Bouvet de la Compagnie de Jésus. A Pékin le 4 novembre $1701 »)$; Nicolas Fréret, légende et vérité, textes réunis et présentés par Chantal GreLl et Catherine Volpilhac-Auger, Oxford, Voltaire Foundation, 1994.

9. Alfred R. Desautels, S.J., Les Mémoires de Trévoux et le mouvement des idées au xvII siècle (1701-1734), Rome, Institutum Historicum Societatis Iesu, 1956. 
l'une des découvertes les plus considérables de l'époque, le calcul infinitésimal ou différentiel ${ }^{10}$. Cette hardiesse des jésuites alors que les académiciens eux-mêmes étaient partagés quant à l'intérêt de cette nouvelle manière d'aborder les mathématiques ne pouvait apparaître comme une preuve de déclin. Il est vrai que Leibniz n'avait pas convaincu le P. Tournemine qui, de plus, s'était montré inquiet devant les suites possibles, sur le plan religieux, de ce calcul. Pendant plus de quarante ans, le P. Louis Castel, chargé, à la rédaction des Mémoires, des mathématiques et de la physique combattit avec acharnement Newton, sans ménager Descartes ni Leibniz. Dans son Vrai système de Physique générale de M. Newton, paru en 1743, il écrivait : «Le Bourgeois Gentilhomme n'est pas le seul qui fasse de la Prose sans le savoir. Les Anciens ont décrit des Ellipses, et les Modernes sont venus deux mille ans après, leur dire que ce qu'ils avoient décrit étoient des Ellipses ${ }^{11}$. " Conclusion : restons-en à Aristote. C'était l'opinion des supérieurs du P. Castel qui, depuis les années 1680 , s'inquiétaient des progrès du cartésianisme parmi les membres de la Société de Jésus. Il n'empêche que l'on débattait longuement dans les Mémoires de Trévoux, parfois avec des défenseurs d'avis opposés, des théories de Leibniz et de Newton, de Maupertuis et d'Euler; qu'on y revenait sans cesse à propos de la publication d'œuvres nouvelles. Les pères, dans leur majorité, pouvaient être réticents et en rester, du moins pour leur enseignement, à Aristote et à saint Thomas. Ils informaient largement, prenaient part au débat et semblaient reconnaître, dans leur for intérieur à partir de 1740, que ce qu'ils s'obstinaient à considérer comme les « hypothèses " de Newton étaient des faits bien établis. Plusieurs événements le montrent. L'un des plus apparents est le zèle dont firent preuve les jésuites mathématiciens et astronomes lors du retour de la comète de Halley en 1759 . On savait que son apparition dans le ciel à la date prévue par le savant anglais serait la meilleure confirmation de la théorie de Newton. Or, ce fut dans les Mémoires de Trévoux de novembre 1757 que Joseph Lalande, confiant dans ses calculs et dans ceux d'Alexis Clairaut, annonça le retour prochain de la comète. Son passage imminent fut signalé dans la même revue au début mars 1759 par les deux académiciens français. Les jésuites, de leur côté, s'étaient mobilisés tant en Chine (Catalogue des comètes vues de Chine du P. Gaubil) qu'en Europe et particulièrement en Allemagne et en

10. Mémoires de Trévoux, mai-juin 1701, p. 223-234 (P. Tournemine); ibid., novembredécembre 1701, p. 270-272 (réponse de Leibniz).

11. R.P. Louis CASTEL, S.J., Le Vrai Système de physique générale de M. Isaac Newton exposé et analysé en parallele avec celui de Descartes, à la portée du commun des physiciens, Paris, 1743, p. 39. 
Europe centrale où de nombreux collèges étaient munis d'observatoires. Ce fut le professeur de mathématiques du collège de Heidelberg, le $\mathrm{P}$. Christian Mayer, qui annonça aux astronomes parisiens Charles Messier et Joseph Nicolas Delisle qu'on avait retrouvé la comète. Celle-ci fut examinée par les mêmes savants, dans la nuit du 31 mars au $1^{\text {er }}$ avril 1759 depuis l'observatoire du collège de Clermont (Louis-le-Grand) ${ }^{12}$. Cette participation des jésuites à l'œuvre scientifique de leur temps n'en resta pas là. Quelques mois plus tard, le professeur de mathématiques du Collège romain, le P. Roger-Joseph Boscovich, présentait, à l'Académie des sciences de Paris, un mémoire sur la Théorie du mouvement des comètes. Dans ce travail, comme dans ceux qu'il avait déjà réalisés, le savant jésuite manifestait clairement son accord avec Newton et son admiration pour sa conception du monde ${ }^{13}$. Ami de Clairaut, Boscovich était aussi membre correspondant de l'Académie des sciences et participait à ses séances lors de ses séjours à Paris.

Certes, l'isolement de ce dernier tant dans le monde savant qu'à l'intérieur de la Compagnie met peut-être davantage en lumière le grand changement opéré depuis le $x^{2}{ }^{e}$ siècle. Le temps où elle était à la pointe du monde savant et intellectuel d'une façon générale était révolu. Mais s'ils n'étaient plus directement impliqués dans la recherche en cours, les jésuites restaient les agents très efficaces de sa diffusion. Ce n'était peut-être pas tant un retrait que la conséquence d'un choix. Au moment où cet ordre missionnaire déployait pleinement son activité dans les villes et les campagnes d'Europe, il ressentait aussi le besoin d'adapter son apostolat aux milieux intellectuels qui, pensait-il, avaient besoin de guides en un temps où toutes les idées et les théories du passé étaient remises en cause. Le journal, beaucoup plus que le collège, était le média qui permettait de guider les réflexions des clercs et des laïcs qui, de plus en plus nombreux, lisaient et réfléchissaient sur les grands problèmes de l'espace, du temps et des origines $^{14}$. On en vient à se demander si les accusations portées contre les jésuites à la hauteur des années 1750 - celles d'être les agents de Rome et de dispenser un enseignement dépassé - ne cachaient pas, chez les hommes des Lumières, une crainte plus réelle. Ils redoutaient peut-être, comme l'écrivait un agent de l'empereur Joseph II à son maître (1773), de

12. Paolo Mafreı, La Comète de Halley. Une révolution scientifique, Paris, Fayard, 1985, p. 312-316.

13. John PAppas, « R. J. Boscovich et l'Académie des sciences de Paris ", Revue d'histoire des sciences, t. XLIX, 4, oct.-déc. 1996, p. 401-414; René TATON, "Les relations entre R. J. Boscovich et Alexis-Claude Clairaut (1759-1764)», ibid., p. 415-458.

14. Sur ce point, la thèse de doctorat d'État de Christian Albertan, Apogée et fin des Mémoires de Trévoux, 1751-1762. Un moment dans la pensée française du xvıf siècle, Paris, université de Paris IV-Sorbonne, 1999, apportera un éclairage nouveau. 
voir les disciples d'Ignace de Loyola «se ménager - sous le voile de la Religion - une influence générale dans toutes les classes du peuple ${ }^{15}$ \%. On était loin d'un déclin.

LOUiS ChÂTELLIER EPHE et IUF, Université de Nancy-2, UFR des sciences historiques et géographiques, 23, bd Albert- ${ }^{\text {er }}$, F-54015 Nancy Cedex.

\section{Luce GiARD}

Réponse aux questions $1,5,6,8$ et 9

Question 1. Par différence avec l'historiographie antérieure, je citerai le déclin des stéréotypes sur l'obéissance inconditionnelle aux ordres (secrets?) reçus du Vatican, ou sur la défense obstinée des énoncés de foi dans leur vérité littérale au détriment des exigences de la Raison et en contradiction avec les avancées des sciences, ainsi que le recul de deux types d'historiographie antijésuite, longtemps majoritaires dans la profession, hors du cercle des historiens catholiques du domaine religieux. Le premier type, encore fidèle aux polémiques des Lumières, aux élans « révolutionnaires $\gg \mathrm{du} \mathrm{XIx}^{\mathrm{e}}$ siècle, ne voyait que des désavantages dans la restauration de la Compagnie et jugeait naturel de transférer à l'histoire de la première Compagnie les traits, conservateurs et obscurantistes, attribués à la seconde. Le second type, producteur d' «histoires nationales ", centrées sur une chronique événementielle du pouvoir politique, de ses institutions et de ses possesseurs, considérait de manière soupçonneuse et défavorable le passé tout entier de la Compagnie, cet ordre religieux international, dont les membres n'étaient pas soumis à la tutelle des épiscopats nationaux, avaient des informateurs un peu partout, se tenaient au courant des choses de ce monde et pouvaient, à tout instant, servir les intérêts d'une nation ennemie ou rivale. Le premier type d'historiographie voyait dans la Compagnie un instrument de contrôle aux mains du pontife et des milieux conservateurs proches de lui. Le second type se méfiait d'une Compagnie incontrôlable par le pouvoir politique national. Les deux s'accordaient sur la noirceur des desseins prêtés aux « hommes en noir ».

Par référence aux plus neuves orientations de l'historiographie contemporaine (la période à prendre en compte serait de dix à vingt ans, selon les

15. Cité par L. Chàtellier, L'Europe des dévots, Paris, Flammarion, 1987, p. 210. 
questions traitées), il est clair que l'historiographie de la Compagnie a grandement bénéficié du renouvellement des problématiques, des intérêts de connaissance et des méthodes dans plusieurs domaines déterminés. Je me bornerai à quelques exemples, dans les secteurs qui me sont plus familiers. Le premier concerne l'histoire intellectuelle prise dans toute son extension, avec l'histoire comparée des systèmes éducatifs, la redistribution des champs de savoir, l'émergence des langues vernaculaires et la place accordée aux littératures nationales à côté de la pratique maintenue des langues classiques et de leur héritage textuel, avec, aussi bien, l'histoire du livre et des bibliothèques, la production des images (livres d'emblèmes, cartes et gravures pour les livres de classe, les manuels de dévotion, les récits venus des terres de mission) et les répertoires symboliques mis au service des idées et des textes. Un second exemple, tout aussi caractéristique, peut être demandé à l'histoire des sciences « nouveau style », devenue inséparable de l'histoire de la circulation et de l'appropriation des savoirs, avec l'accent mis sur le rôle moteur de micromilieux actifs et organisés, avec l'importance accordée moins au génie des " grands hommes " qu'à leur insertion stratégique dans des réseaux vivants de production et de circulation des savoirs (correspondants, commentateurs, traducteurs, éditeurs, etc.), milieux et réseaux associant leurs efforts et rivalisant dans la mise à l'épreuve des théories et de leurs pratiques cognitives, comme dans leur appropriation et leur diffusion. Une manière plus nuancée et plus complexe de se représenter les modalités de changement dans les savoirs a rendu aux jésuites, à côté de leur rôle familier d'éducateurs, une fonction essentielle de «circulateurs » intellectuels dans l'émergence de la modernité.

Une autre branche, dont l'essor a bénéficié à l'historiographie de la Compagnie, concerne la sortie de l'Europe hors de ses frontières, la formation des empires coloniaux, le développement des missions outre-mer. À côté d'une histoire économique et politique aux formes anciennes sont apparues, conjointement, dans une dialectique d'antagonismes et de complémentation réciproque, une histoire anthropologique de la rencontre de l'autre (autres formes de sociétés, de religions, de cultures et d'usages) et une géopolitique marquée par les conflits et les lignes de partage de la Guerre froide et des multiples guerres d'indépendance. Il se trouve que les jésuites d'autrefois avaient souvent été des observateurs attentifs et perspicaces de leurs interlocuteurs d'Amérique ou d'Asie, et que leurs observations subsistent dans d'innombrables documents d'archives, dans les extraits publiés dans les Lettres édifiantes et curieuses, dans des livres ayant diversement circulé. Il se trouve également que les jésuites de ce dernier demi-siècle ont souvent marqué leurs distances avec les pouvoirs en place et peu à peu rompu les liens d'identification (fondés ou non, dans la 
réalité des situations) qui les associaient antérieurement aux milieux conservateurs de leurs anciens territoires de mission. Les deux effets cumulés ont suscité des travaux neufs sur leur action hors d'Europe, dans d'autres domaines que la seule histoire religieuse.

Le renouveau de l'historiographie de la Compagnie est venu de la conjonction de tous ces éléments. Dès lors, l'histoire de la Compagnie ou de certains jésuites dans certains lieux (sociaux, géographiques, intellectuels, politiques) est devenue digne d'intérêt au-delà des frontières du domaine religieux proprement dit. La première conséquence a été un double élargissement : élargissement du milieu de recrutement des historiens s'occupant, peu ou prou, de la Compagnie; élargissement des perspectives inspirant leurs travaux. En ce sens, l'histoire de la Compagnie désormais n'est plus un domaine réservé soit aux jésuites devenus historiens de leur propre tradition (mais leur rôle est encore essentiel dans la publication des sources, des inventaires et des histoires par « provinces jésuites »), soit aux historiens de l'Église et du catholicisme (à travers tout l'éventail de ses manifestations et pratiques). Par là, l'histoire de la Compagnie tout entière a bénéficié d'un « désenclavement » décisif et pleinement significatif. Ce désenclavement a porté tout autant sur l'invention des sources, leur analyse comparée avec des documents contemporains issus d'autres milieux, que sur la formulation de nouvelles interrogations, l'essai d'autres problématiques, le recours à des méthodes inspirées par d'autres sciences sociales. Ainsi la production jésuite des livres d'emblèmes, dont on mesure de mieux en mieux l'extraordinaire abondance, n'a plus seulement été considérée comme une pratique de dévotion ou d'endoctrinement (selon le cadre d'interprétation retenu), mais on a commencé à la mettre en rapport plus largement avec l'histoire du livre et des cultures de cour, avec celle des décors intérieurs d'églises, avec les répertoires d'iconographie et les affixiones composées par les élèves des collèges pour célébrer des solennités particulières, avec la pratique théâtrale, développée à grande échelle dans les collèges. À son tour, le théâtre jésuite, sans cesser d'être vu comme une pratique d'édification religieuse et de transmission active, dans ses formes littéraires et ses thèmes narratifs, de l'héritage antique grec et latin, est aussi devenu un lieu d'apprentissage et de codification de la mise en scène des corps et de l'expression des liens sociaux, mais on y reconnaît tout autant le lieu d'expérimentation d'une rhétorique et d'une stylistique des langues, anciennes et modernes, un travail mené à un rythme et d'après des canons différents dans des contextes linguistiques et nationaux distincts. Que des travaux récents reprennent la vieille question du milieu religieux et culturel auquel appartenait le jeune Shakespeare, que certains historiens semblent aujourd'hui enclins à prouver qu'il fut bien, pour un temps, membre d'un réseau de courriers et de jeunes accompagna- 
teurs des jésuites circulant, célébrant et prêchant incognito en Angleterre, que le silence ensuite gardé sur ses années de jeunesse fut destiné à effacer ces liens dangereux, tout cela ne fait qu'ajouter un grain de sel à un plat déjà consistant, c'est-à-dire ne peut qu'encourager à reprendre divers épisodes de l'histoire de la première Compagnie en des termes nouveaux.

Question 5. Par rapport aux ordres antérieurs, la première spécificité de la Compagnie, la plus significative pour expliquer la particularité de son devenir, est certainement sa « jeunesse ». N'ayant ni à défendre la fidélité à une tradition antérieure, ni à craindre les conditions nouvelles de la vie sociale au $\mathrm{XvI}^{\mathrm{e}}$ siècle, la Compagnie put dépenser toute son énergie et son talent (l'un et l'autre furent grands) à chercher à comprendre la novation du monde contemporain (luttes confessionnelles, transformations économiques et sociales, théorisation du pouvoir politique et des liens de subordination à son égard, demande d'éducation, circulation du livre imprimé, relecture de « l'histoire du salut » imposée par l'expansion européenne en Amérique et en Asie). Sur tous ces points, il serait faux de créditer la Compagnie d'une totale lucidité ou d'un parfait discernement dans l'invention des pratiques et des interprétations théoriques, mais on peut saluer la conscience aiguë qu'elle eut, dans son ensemble, de l'urgence des problèmes, de leur importance, de la nécessité d'y répondre, comme on peut saluer la constance de ses efforts pour tenter d'y apporter des solutions de fond, l'intelligence d'une réflexion attentive aux conséquences à long terme et à grande échelle.

Ici, on touche à sa seconde spécificité, qui relève des effets d'échelle. Son rapide essor quantitatif (mesurable par le nombre d'entrées, de fondations de collèges, de groupes envoyés dans les missions d'outre-mer), son succès (visible dans la réputation gagnée comme éducateurs, confesseurs èt conseillers des princes, et dans l'opposition tenace d'autres ordres, ou la multiplication de ses ennemis politiques) et la dispersion géographique de ses membres lui ont permis de ne pas s'enfermer dans les horizons de la vieille Europe. L'un et l'autre ont nourri son désir constitutif (voir les Constitutions) d'adaptation au local et au circonstanciel. Ainsi a-t-elle pu créer une figure nouvelle de religieux, consacré à Dieu et tourné vers la modernité, inventif et mobile, actif et réfléchi, souple et ferme, tous ses traits spécifiques étant incarnés avec plus ou moins de bonheur et d'audace selon le talent et les responsabilités de chacun. Dans l'organisation de la Compagnie comme institution, l'accent, fondateur et régulateur, placé sur le maintien de l'unité, dans la dispersion des lieux et la variété des tâches, a conduit à l'invention d'un instrument propre à entretenir cette unité. Ignace et son secrétaire Juan Alfonso de Polanco trouvèrent cet instrument dans la mise en place d'un système général de correspondances régulières et détaillées, circulant à tous les échelons, traitant de tous les sujets, maintenant le 
gouvernement central de l'Ordre informé des réalités de chaque communauté et faisant retourner, sous forme condensée, l'essentiel de ces informations et nouvelles à tous les compagnons. En cela, la Compagnie fut organisée comme un immense réseau de communication et parvint à le demeurer à travers les vicissitudes de son histoire. Exerçant les capacités d'observation et d'analyse des jésuites (la rotation des charges et des responsabilités y contribua aussi), ces multiples correspondances ont élargi les perspectives, aiguisé la conscience politique et sociale des changements survenus dans le monde alentour, nourri un incessant débat d'idées. Bien sûr, celui-ci ne fut pas toujours irénique et positif, il fut accompagné d'innombrables conflits d'opinion (sans compter les rivalités et inimitiés entre les individus, souvent associées à des rivalités entre groupes nationaux dont les objectifs d'apostolat et les idéaux de piété pouvaient différer notablement), marqués par toute sorte de désaveu (lettres de dénonciation au Supérieur général, censure des livres, assignation à d'autres tâches moins valorisantes ou moins exposées publiquement). Mais cet incessant débat d'idées eut aussi le mérite de faire penser en termes géopolitiques, de faire quitter l'ici et le maintenant pour un ailleurs du temps à venir.

Cette organisation particulière de l'institution n'a pu être efficace et durable que grâce à une spiritualité adéquate, tout aussi " moderne », prônant un engagement résolu dans l'action en ce monde-ci, un engagement politique et mystique, ayant pour objectif la transformation et la mobilisation vertueuse des personnes et des groupes, le tout appuyé sur une confiance joyeuse dans la signification de ce qui était à faire et qui serait accompli ad majorem Dei gloriam. De cette confiance agissante, un trait essentiel fut le respect continu manifesté pour le travail de l'esprit, pour ses questions, pour le désir de savoir (dont Aristote avait fait un caractère définitoire de la nature humaine). D'où est né ce que j'ai appelé ailleurs le « devoir d'intelligence » que la Compagnie, dès ses commencements, s'est donné à elle-même. Ce fut son honneur et sa grandeur d'avoir toujours cherché à y répondre en différents registres et par diverses voies : fondation des collèges, jésuites libérés de toute autre tâche pour se consacrer au débat d'idées et à l'écriture, "veille » maintenue sur le front de la recherche en philosophie comme en sciences ou en théologie. D'autres ordres religieux, antérieurs ou contemporains, ont, dans la même période, donné à l'Église de grands intellectuels, des hommes de savoir et d'étude, mais aucun autre ordre ne s'est pareillement identifié tout entier au service des intelligences.

Question 6. C'est un fait. À mesure qu'elle a avancé en âge et en pouvoir, il semble que la Compagnie a perdu en inventivité, en audace et en liberté de mouvement. Est-elle alors devenue trop proche des structures de pouvoir politique et social, a-t-elle perdu son élan premier ou son discernement sur la société autour d'elle? Ou serait-ce une image fausse, venue de 
notre propre ignorance de son histoire après 1650 ? Quelques récentes recherches (Marcus Hellyer sur la physique dans les collèges d'Allemagne aux $\mathrm{XVII}^{\mathrm{e}}$ et $\mathrm{XVII}{ }^{\mathrm{e}}$ siècles ${ }^{16}$, d'autres dont les premiers résultats ont été présentés au colloque jésuite de Boston en mai $1997^{17}$ ) ont montré que les choses n'étaient ni aussi simples ni si nettement tranchées. Comme historiens, nous avons tout à gagner à voir les travaux de première main se multiplier sur l'histoire de la Compagnie dans le second XVII siècle et le XVIII' Sur les problèmes spécifiques concernant la seconde Compagnie, je ne me prononcerai pas, en raison de mon incompétence en la matière.

Question 8. Je n'entends pas faire ici l'apologie de ma manière d'étudier l'histoire des jésuites. Je suis venue à m'y intéresser pour deux raisons : leur présence dans le dêbat philosophique et scientifique au tournant des $\mathrm{XVI}^{\mathrm{e}}$ et $\mathrm{XVII}^{\mathrm{c}}$ siècles, leur caractère international (qui conservait un aspect de l'université médiévale, tout en l'inscrivant en termes nouveaux dans l'espace public et dans leur propre système éducatif). J'ai essayé de traiter de leur histoire en la « désenclavant » de l'histoire religieuse, pour la placer dans une histoire intellectuelle élargie et enrichie. Mais, en même temps, j'ai voulu très consciemment rendre justice à la spécificité de l'entreprise ignatienne (spiritualité, objectifs et modes d'action). Autrement dit, j'ai cherché à ne pas enfermer les jésuites de la Renaissance (ma période d'étude) dans un rôle stéréotypé et, en même temps, à comprendre ce que leur appartenance volontaire à la Compagnie apportait de coloration propre à leur situation de pensée. Pour reprendre un vocabulaire familier aux historiens des sciences, dans un autre cadre, $j$ 'ai essayé, à propos de la première Compagnie, de nouer une histoire internaliste à une histoire externaliste. Dans cette intention, j'ai saisi les occasions qui se sont présentées de travailler sur le corpus ignatien lui-même : Lettres et instructions, Constitutions (résultats déjà publiés dans les deux cas) et Exercices spirituels (étude en cours). Cet aspect de l'identité jésuite me semble essentiel. Faute d'y réfléchir et d'acquérir quelque familiarité avec cette tradition spirituelle, on se condamne à une description superficielle et à une compréhension limitée de l'histoire intellectuelle des jésuites auxquels on s'intéresse dans un domaine particulier du savoir. Je suggère donc que, pour « désenclaver » l'histoire de la Compagnie, l'historien consente d'abord à "désenclaver » son propre esprit et à traverser les frontières entre branches de sa discipline. J'ai tendance à croire qu'il y a tout à gagner dans cette sorte de voyage intellectuel. Mais je reconnais qu'il a son coût.

16. Voir, dans la présentation de ce volume, la référence à sa thèse, p. 254, n. 25.

17. Voir le dossier bibliographique sur l'histoire générale de la Compagnie de Jésus, p. 433-439. 
Question 9. En termes chronologiques, le plus nécessaire serait d'approfondir notre connaissance de la Compagnie, entre 1650 et sa suppression. En termes d'objets, je soulignerais ce qui relève d'une histoire économique (ressources, engagement dans la gestion des biens et certains types de commerce, recours outre-mer au travail d'esclaves et conduite à leur égard) et des rapports diversifiés (selon les régions et les groupes sociaux) avec le monde non-européen. Dans l'espace européen, il faudra bien aborder en face, à partir des sources, l'articulation entre philosophie, sciences et théologie, car certaines décisions sur les théories en tel domaine ne sont compréhensibles qu'en relation avec leurs conséquences, réelles ou supposées, dans le domaine voisin. Rendre toute son importance et sa vivacité au débat théologique dans la première Compagnie (pour elle comme pour le monde qui l'entoure) nous permettra de mieux comprendre les aléas de notre première modernité et d'approfondir, en particulier, notre analyse du débat théologico-politique (voir les travaux prometteurs de Sylvio De Franceschi à ce propos ${ }^{18}$ ). On peut espérer que le temps est venu de se soustraire à la fixation sur le cas Galilée et d'élargir notre champ d'exploration et de réflexion.

Luce GIARD

CNRS/CRH, EHESS,

54, bd Raspail, F-75006 Paris.

\section{Dominique JuLIA}

Réponse aux questions $1,2,3,4,5,6,7$ et 9

Question 1. Parmi les traits saillants du renouvellement de l'histoire de la Compagnie de Jésus, je retiendrais d'abord l'abandon des histoires «nationales» de la Compagnie qui saisissent l'activité de celle-ci dans chaque pays, même si ce type d'histoire se poursuit encore pour la péninsule italienne. Il est vrai que, dans ce cas précis, l'histoire de la Compagnie se relie plus directement au centre romain et aux directives des différents généraux, et dépasse le cadre étatique dans une péninsule morcelée en

18. Sylvio De Franceschi, La France et l'interdit vénitien, 1606-1607. Aspects diplomatiques et doctrinaux, DEA, Paris, École pratique des hautes études, IV ${ }^{\mathrm{e}}$ section, 1998; ID., Antiromanisme doctrinal, pouvoir pastoral et raison du prince. Le prisme français (16061611), thèse pour l'obtention du diplôme d'archiviste paléographe, Paris, 1999. 
diverses entités politiques. Ces histoires " nationales » qui ont connu leur apogée à la fin du $\mathrm{XIX}^{\mathrm{e}}$ siècle et dans la première moitié du $\mathrm{Xx}^{\mathrm{e}}$ siècle ont effectivement correspondu à la nationalisation des historiographies pour cette période mais avaient l'inconvénient de perdre de vue les logiques à l'œuvre dans les décisions romaines, en particulier la vision au moins européenne, sinon planétaire, qui fonde l'action du Général. La volonté de prendre à bras le corps certaines questions, ainsi les missions, de manière «transcontinentale », permet au contraire de mesurer les convergences comme les décalages ou les dénivellations qui s'opèrent entre les différents pays et de saisir les raisons qui peuvent les expliquer. Un deuxième aspect de ce renouvellement est que les interrogations portées sur la Compagnie de Jésus partent moins de l'histoire proprement religieuse que des problématiques développées dans les différents champs de l'historiographie : l'intérêt que la sociologie des sciences manifeste à l'égard des raisons pour lesquelles un milieu précis s'attache à développer des travaux scientifiques fait redécouvrir la Compagnie comme productrice de savoirs et participant à la sociabilité scientifique de l'époque moderne (académies, correspondances scientifiques, observatoires, etc.); les interrogations que la sociologie scolaire porte sur les facteurs qui règlent la réussite ou l'échec scolaire renvoient aux fonctions qu'exerçaient les collèges de la Compagnie dans la société de leur temps; la question de l'acculturation et de la destructuration des sociétés indiennes est au cœur de la relecture des archives relatives aux missions. Le renouvellement de l'histoire de la Compagnie passe par les reconfigurations des problématiques dans chaque champ de l'historiographie, ce qui modifie le regard posé sur les textes.

Question 2. Au sein de l'analyse historique du phénomène religieux, l'histoire de la Compagnie de Jésus s'est attachée à préciser les stratégies que celle-ci a développées pour canaliser et modeler la piété dans les diverses couches de la société : on n'est pas éloigné ici de la problématique de la Disziplinierung ou du disciplinamento. Les congrégations mariales que la Compagnie dirige et qui encadrent les diverses catégories sociales dans les villes (nobles, marchands, bourgeois, artisans) visent à fournir un règlement de vie aux laïcs conformément à leur état, en rythmant leur emploi du temps par des exercices, en contrôlant les esprits, les corps et les cœurs. L'histoire des missions populaires dans les « Indes de l'intérieur » a étudié la pédagogie à l'œuvre dans les prédications, l'importance qu'y prend la mise en scène offrant des spectacles frappant la foi des rudes pour mobiliser les différentes facultés de l'âme, l'acculturation qu'y opèrent les missionnaires en reprenant rites, calendrier des fêtes, processions et en remodelant gestes et pratiques dans le sens d'une orthopraxie. On pourrait rattacher à cette rubrique l'étude de la direction de conscience exercée par les jésuites auprès des élites et tout particulièrement la tâche dévolue au 
confesseur du prince, à cette frontière essentielle et mouvante du religieux et du politique. En même temps, et plus globalement, l'étude de la Compagnie a montré que celle-ci est présente souvent aux lieux et aux moments où se dévoilent des mutations culturelles décisives de l'époque moderne : ainsi lors des phénomènes de possession, théâtre devenu si étrange à nos yeux. Alors que les commissaires du roi récupèrent sous le joug de la loi politique les rébellions diaboliques appelées à devenir autant de «cas royaux ", et mobilisent le sacré au service de l'État, les jésuites impliqués - ainsi Jean-Joseph Surin - se comportent moins en exorcistes au service du pouvoir qu'en directeurs spirituels voulant travailler les âmes par la voie " intérieure ", entreprise qui ne laisse pas indemne celui qui s'y engage. Indice qui renvoie à un problème plus général traversant l'ensemble du corps : comment une institution religieuse peut-elle préserver son « intériorité », son esprit, alors que la sécularisation des travaux, des méthodes et des pratiques, l'effusio ad exteriora ne cessent de la menacer. D'où la volonté de restaurer cet esprit par une organisation juridique et intellectuelle qui le fasse tenir: réglementation des pratiques spirituelles, des études (Ratio studiorum), production d'une littérature spirituelle destinée à articuler la hiérarchisation des tâches au fondement qui les légitime. En ce sens, la Compagnie est bien, à la fin du $\mathrm{XvI}^{\mathrm{e}}$ et au début du Xvil ${ }^{\mathrm{e}}$ siècle, traversée par une «politique de la spiritualité » (Michel de Certeau), qui vise à restituer une cohésion en péril.

Question 3. Le renouvellement de l'histoire de la Compagnie est d'autant plus important que celle-ci a fourni une bonne partie des élites dans l'espace de la catholicité. C'est donc le remodelage de l'héritage intellectuel auquel a procédé la Compagnie pour sa pratique pédagogique qu'il s'agit d'interroger pour saisir quels habitus culturels la Compagnie a enracinés à chaque époque de son histoire chez ses élèves. Ce qu'il convient de retrouver, ce sont les manières de procéder, les types d'apprentissage intellectuels (par exemple, formation à la copia et aux lieux communs en rhétorique) pour comprendre les mécanismes de pensée de l'homme moderne. En réalité, le renouvellement de l'histoire de la Compagnie touche tous les champs de l'histoire moderne, de l'histoire des sciences (mathématiques, physique, géographie, etc.) à celle des arts (architecture, théâtre, musique), de la pensée politique (ainsi Giovanni Botero qui fut un temps jésuite) ou du rapport de l'Europe avec les autres civilisations (avec les missions et tout particulièrement les missions de Chine, d'Inde ou d'Amérique latine).

Question 4. Le temps est sans doute lointain - c'était en 1858 - où un archiviste, comme celui de la ville de Dôle, rédigeant la description d'une liasse où il notait « deux cahiers où sont inscrites les admissions de novices en la maison de Dôle et plusieurs pièces relatives aux vœux et professions 
religieuses des Frères » des Écoles chrétiennes concluait : « En somme, ce recueil offre peu d'intérêt. » Tout le travail qui a porté sur les ordres et les congrégations religieuses s'est au contraire attaché à reconstruire les courbes d'entrée dans les noviciats ou maisons d'institutions pour saisir le sens du choix de religion au sein des diverses catégories sociales à l'époque moderne, qu'il s'agisse des ordres anciens ou des congrégations nouvelles. Au-delà de cette histoire sociale nécessaire mais insuffisante, les historiens ont cherché à comprendre comment les créations d'ordres religieux nouveaux (ou les réformes d'anciens) constituaient, pour reprendre la formulation de Jean Séguy, des «utopies pratiquées » contestant un état présent de l'Église en cherchant à remettre en cause certains aspects de la pratique actuelle par l'exemplarité dont est porteuse la nouvelle société monastique. Ce sont les historiens médiévistes qui se sont davantage posé ce type de questions, notamment à propos des franciscains et des fractures successives qui ont progressivement décomposé l'ordre en diverses branches. C'est ici que la confrontation entre les textes fondateurs et le fonctionnement réel des instituts créés peut se révéler particulièrement féconde.

Question 5. Les spécificités propres à la Compagnie de Jésus par rapport aux ordres contemporains sont nombreuses et elles ont justement provoqué des incompréhensions et des réactions extrêmement vives. La première est la visée universelle de la Compagnie dont la mission - « se consacrer au bien des âmes dans la vie et la doctrine chrétiennes et à la propagation de la foi » - s'étend au monde entier, les jésuites se plaçant sous l'autorité du souverain pontife par un vœu spécial et s'engageant : " [à se rendre] sans aucune tergiversation ni excuse, immédiatement, autant que cela dépend de nous, soit qu'il nous envoie chez les Turcs, soit au nouveau monde, soit chez les luthériens, soit chez n'importe quels autres infidèles ou fidèles " (Summa de 1539). C'est cette vocation universelle, liée directement à l'expansion du monde connu par les découvertes qui, en retour, a déclenché la constitution du mythe jésuite comme État tentaculaire, se dressant, par l'obéissance qu'il requiert de ses membres vis-à-vis du général, contre les États modernes. Une seconde spécificité tient au refus d'une série de règles qui apparaissent liées de manière essentielle à la vie religieuse : absence d'office choral et d'orgues, refus d'un habit spécifique, absence de jeûnes et de mortifications corporelles, vie au sein du monde pour le transformer. L'œuvre à accomplir est l'instauration du christianisme chez tous les hommes, clercs ou laïcs, et comptent davantage ici les dispositions intérieures des membres, leur ascèse qu'une clôture matérielle. D'où la troisième spécificité de la Compagnie qui réside dans la longueur de la formation. Alors que le temps d'épreuve dans les autres ordres ou congrégations religieuses est le plus souvent limité à un an ou deux, la longueur de la for- 
mation et le rapport inégal qui s'introduit entre la Compagnie et ceux qui sont reçus avant leur profession définitive - la Compagnie restant à tout moment libre de renvoyer - distinguent nettement le nouvel ordre de tous les ordres religieux antécédents, de même que les « expériences » qui précèdent l'incorporation (service des hôpitaux, pèlerinage, exercices spirituels). Enfin, l'usage de la correspondance à l'intérieur de la Compagnie, qui est l'un des moyens spécifiques voulus par Ignace pour maintenir l'ensemble du corps dans un même esprit, fait l'originalité de cette société. Si cette richesse de la correspondance, dont les règles ont été fixées très tôt (cf. la lettre à Pierre Favre du 10 décembre 1542, ou la lettre à toute la Compagnie du 27 juillet 1547), fait aujourd'hui le bonheur des historiens, il ne faut jamais oublier que cette correspondance a pour but « la gloire divine et l'édification du prochain ". D'une certaine façon, l'union des membres dispersés dans le monde par la correspondance se substitue à l'ancien opus divinum des communautés monastiques. On comprend que tous ces traits (et bien d'autres) aient pu être difficiles à saisir d'emblée. Le visiteur du Portugal Miguel de Torres n'exprime-t-il pas à Ignace de Loyola en août 1553 son désir de voyager avec le P. Jerónimo Nadal non seulement pour le servir, mais « pour apprendre avec lui le mode [de procéder] de la Compagnie parce qu'à vrai dire, Père, je ne le comprends pas et même je ne sais pas bien comme il convient la fin de la Compagnie »?

Question 6. Trois raisons, entre autres, me paraissent expliquer le privilège accordé au premier siècle de la Compagnie. La première - qui est généralisable à l'ensemble des ordres religieux - est liée à la recherche des origines qui a saisi l'ensemble de ceux-ci à partir des années 19501960 dans un mouvement concomitant à celui du concile Vatican II. Toute réforme passait par un retour obligé aux sources, une relecture attentive du moment de la fondation et des premières étapes de l'Ordre. C'est ainsi au moins qu'on peut interpréter le développement de la collection Christus aux éditions Desclée De Brouwer ou l'édition des Monumenta paedagogica (qui démarre en 1965 et en est aujourd'hui à son septième volume). En second lieu, le récent $450^{\circ}$ anniversaire de la fondation de la Compagnie (1990) qui coïncidait quasiment avec le cinquième centenaire de la naissance d'Ignace de Loyola (1991) ont donné lieu à toute une série de colloques, livres collectifs, rééditions critiques de textes fondateurs (ainsi les Écrits d'Ignace de Loyola, traduits et présentés sous la direction de Maurice Giuliani), qui ont privilégié comme angle d'approche la seconde moitié du xvI siècle et la première moitié du $\mathrm{xvII}^{\mathrm{e}}$ siècle. Enfin, il est clair que la confrontation des textes essentiels (Exercices spirituels, Constitutions, et Règles, Ratio studiorum) avec les pratiques réelles des premiers jésuites telles que les livrent les correspondances conservées offrait l'opportunité de saisir un idéal de vie religieuse en acte dans ses diverses potentialités 
dispersées à travers le monde. La proximité temporelle des uns aux autres exerçait une force d'attraction aisément compréhensible pour qui veut comprendre le fonctionnement d'un modèle.

Le désintérêt pour le second $x{ }^{e}{ }^{e}$ siècle et le xvIII ${ }^{e}$ siècle me paraît partir d'un double préjugé selon lequel, d'une part, les jésuites auraient dès lors cessé d'appartenir à la "modernité » en privilégiant dans leur enseignement les humanités latines et en refusant d'y intégrer les novations scientifiques, et, d'autre part, qu'ils seraient entièrement absorbés, sur le plan théologique, par leur lutte antijanséniste. Il faudrait très certainement faire le même travail critique pour cette période que celui qui a déjà été accompli pour la première période en sortant des sentiers battus. J'ajoute que, contrairement à ce qui a été fait pour d'autres ordres religieux, l'étude du personnel de la Compagnie n'a pas donné lieu (en dehors des études d'Adrien Demoustier sur les catalogues jésuites français à la fin du Xvie siècle et au début du Xvil ${ }^{e}$ siècle et de Thomas Cohen sur les jésuites espagnols du Xvi ${ }^{e}$ siècle) à de très nombreux travaux. Il serait pourtant capital, pour une meilleure compréhension de l'action de la Compagnie, de saisir les liens familiaux qui peuvent unir les membres aux différentes élites des divers royaumes ou principautés en Europe. En dépit des travaux qui ont été pionniers (ainsi ceux de François de Dainville), il n'est pas sûr que nous soyons complètement sortis de l'historiographie polémique qui opposait au début de ce siècle les historiens jésuites aux réquisitoires rédigés par les historiens attachés aux Lumières et dénonçant l'obscurantisme d'un ordre inféodé à un général étranger...

Question 7. La redécouverte d'un espace « européen " de la Compagnie rend pleinement son rôle au centre d'impulsion romain et restitue un type de cohérence plus pertinent à l'action des jésuites que les histoires « nationales " qui partaient d'un principe que l'on pourrait appeler davantage "gallican". Il ne s'agit aucunement de nier les évolutions divergentes qui ont pu se produire entre les différentes provinces. Mais ce sont désormais ces divergences, ces décalages, ces dénivellations dont il faut pouvoir rendre compte dans leur rapport à l'autorité romaine.

Question 8. Refus de répondre.

Question 9. La prospective n'est pas la qualité primordiale de l'historien : il risque de prendre ses désirs pour des réalités à venir, alors que, comme dans toute historiographie (et peut-être plus dans celle-ci que dans toute autre), les inerties liées à une longue tradition peuvent peser sur l'orientation des travaux. On peut citer au moins deux chantiers qui pourraient être approfondis dans les prochaines années. Le premier serait une analyse plus systématique du travail des censeurs à l'intérieur de la Compa- 
gnie et des censures qu'ils ont produites concernant les ouvrages soumis à leur jugement. Il serait possible ici de saisir le fonctionnement de l'orthodoxie au sein d'un ordre religieux, ses mécanismes, ses critères d'appréciation, les freins qui ont pu ainsi être mis au développement des expériences scientifiques, ou au contraire les portes laissées ouvertes. Le second chantier, lié à l'ouverture des archives de la Congrégation pour la doctrine de la foi, pourrait être d'examiner le rôle qu'ont pu jouer les jésuites (notamment les consulteurs) dans les décisions doctrinales soumises à l'autorité de la Congrégation. Si des études nombreuses ont déjà éclairé en partie ces questions (il suffit de songer aux travaux du P. Lucien Ceyssens ou de Henk Hillenaar), il est probable que les dossiers désormais ouverts apporteront, là aussi, des éléments tout à fait neufs sur le processus d'orthodoxie et ses enjeux comme sur la participation des jésuites à celui-ci.

Ce qu'il convient d'atteindre en tout cas, c'est, au-delà des stratégies d'écriture qui caractérisent les textes jésuites, les pratiques réelles auxquelles se sont livrés les membres de la Compagnie (ainsi dans l'enseignement par l'étude des manuels et des copies d'élèves, dans les missions par l'écart qui sépare les informations reçues des missionnaires et restées manuscrites des lettres réécrites et publiées par les jésuites européens pour un public qu'il s'agit d'édifier, etc.). La remarque vaut pour l'ensemble des secteurs de l'activité apostolique de la Compagnie.

\author{
Dominique JuLIA \\ CNRS/CARE, \\ EHESS, \\ 54, bd Raspail, \\ F-75006 Paris.
}

\title{
John O'MaLLeY
}

Réponse aux questions $1,3,4,5,6,7,8,9$

In what follows I will speak principally from the perspective of Englishlanguage historiography.

Question 1. Three traits of the new interest in the Society of Jesus are striking and fairly obvious. First, until quite recently serious research and publication of sources were done almost exclusively by Jesuits. That situation has now changed radically. The second trait is the new prestige and attention given to the history of the Society outside Europe, with a concomitant effort to integrate it with European history. The third is a notable diminution of both the traditional apology and polemic. 
Question 2. In dealing with religion, anglophone historiography has been dominated by the Reformation, with the Catholic phenomenon at best an afterthought, dull. That has begun to change. All at once early modern Catholicism is interesting! Moreover, new categories of interpretation, such as " confessionalization " and " social disciplining ", sometimes seem poised almost to swamp more traditional categories like reform, Reformation, and Counter-Reformation. An advantage of these new categories is their applicability to both Protestant and Catholic phenomena, thus underlining the profound similarities between them.

A disadvantage is that under new forms they continue a historiographical tradition of studying religion for its social or political impact and thus seem incapable of dealing with it as an expression of the sacred. Religion is reduced to a will to power, a ploy to domination. Despite the efforts to take account of all disciplines in order to construct a « total history », religious sentiment and spirituality are still not given serious consideration on their own terms. The Jesuits are studied, often sympathetically, as clever, even «modern » people. But their heart and soul is missing. I make an exception for art historians, some of whom successfully overcome this dichotomy.

Question 3. In anglophone historiography the current surge of interest in the Jesuits is too recent to have had great impact. It has, however, already functioned as an important reminder that not everybody in the sixteenth century became a Protestant. Besides helping to break down traditional Eurocentricity, it has great potential for fostering a more interdisciplinary approach to the history of the modern world, for an individual Jesuit might be some combination of architect, musician, missionary, ethnographer, political philosopher, and so forth.

Question 4. One of the great fruits of the integration of the history of religious orders into general history would be an assault on the deep-seated framework of interpretation that identifies Catholicism with « the Catholic Church ", viz., with pope, bishops, pastors of parishes, and, perhaps, with dissident or " oppressed » laity. In such a scheme the religious orders, both male and female, have no role. Historians often betray this prejudice. They can hardly be persuaded, for instance, that the bull of Pope Paul III establishing the Society of Jesus was simply a charter allowing the Society to operate and not an incorporation of it into the Roman Curia! The history of the female compagnies of seventeenth-century France adds of course a fascinating dimension to women's history in the early modern era, and many of those compagnies took their inspiration from the Society. 
Question 5. The Society of Jesus was special in a number of ways but two are of overwhelming importance. No order had a book like the Spiritual Exercises, which not only expressed a certain spirituality but also provided a process for interiorizing religious ideals. In this regard the Exercises manifested a break with the observantist tradition in religious life, which is much older than the late Middle Ages. I believe that the singularity of this feature of the Society has never been fully comprehended by historians. The Exercises allowed, therefore, for Constitutions that were not, as in the other orders, a collection of ordinances, but an expression of spiritual wisdom that would rely for successful implementation on the spiritual discretion of the superior.

The second is the schools, which gave the Society a unique, newly systemic relationship to culture. Here the humanistic curriculum was of special importance, for it forced every Jesuit to become a professional in « pagan » culture - in order to teach it.

The "philosophical " component in the curriculum forced them to become teachers of science and mathematics. This was, as a system, unprecedented for a religious order. True, other orders later began to staff and manage schools, following the Jesuit example, but, even so, none had an institution in any way comparable in magnitude and sophistication to the Collegio romano (as well as other schools), which the Society sustained and fostered over the course of two centuries.

Question 6. With the exception of the Annalistes, historians have always been more interested in origins than in durations. For a religious order, origins have always been considered crucially important, for they are assumed to be the fons et origo of the charism of the group, and the founding members are assumed to be models of sentiment and deportment for all time. With the Jesuits these tendencies were exacerbated by a historiography that made Saint Ignatius an emblematic counterpart for the Catholics to what Luther was for the Protestants.

The "substantialism » that characterized much of Catholic historiography also made later history less interesting, for it was considered merely a further expression of what was present in the origins. Little account was taken of how profoundly institutions change in the course of time, even when similar-seeming formalities are still in place. If we are to understand the Jesuits, we of course must move beyond the first generation. That is the task that lies ahead, but it is already being addressed by many scholars throughout the world.

Question 7. Just as we today see more similarity between sixteenthcentury Protestants and Catholics than differences, so we see more similari- 
ties than differences between Jesuits coming from different « nations». But the regional differences must not be minimized!

Question 8 . I began to study the Jesuits only after many years studying other aspects of the religious culture of early modern Europe. I believe this has allowed me to place the Jesuits in a larger cultural context than earlier scholarship, much of which was done in a cultural vacuum. I have also tried to provide an integrated approach, taking account of as many aspects of their ideals and activities as possible. I have tried to ground my approach in their ministries, for « the help of souls " was the reason they were founded. I have tried to correlate their normative documents (Constitutions, « Rules », « Letter on obedience », etc.) with their actual practice. That has meant, finally, that I have tried to show what their religious motivation (or " spirituality ") was, how it operated, how it was sometimes compromised, how it changed. One of the official "readers " for Harvard University Press of my book on the Jesuits said it was about spirituality. This suprised but pleased me.

Question 9. I propose two big tasks. The first is synchronic: up to this point, the Society and individual Jesuits have, as I suggested above, been studied in too compartmentalized a fashion. How at any given time and place are spirituality, music, preaching, schools, religious polemics, and so forth integrated so as to provide a coherent and comprehensive picture? The second is diachronic: we must trace the changes that occurred over time. In my First Jesuits I tried to show four stages of change between 1534 and 1565 . What were the major stages afterwards, even up to the present?

John O'MALLEY, S.J. Weston Jesuit School of Theology, Cambridge, MA, 